\title{
Chirurgische Ausbildung im digitalen Zeitalter - Virtual Reality, Augmented Reality und Robotik im Medizinstudium
}

\author{
Surgical Education in the Digital Age - Virtual Reality, \\ Augmented Reality and Robotics in the Medical School
}

(c) (1) $\ominus$

\author{
Autoren \\ Institute \\ 1 AG 4 - Digitale Medizin, Medizinische Fakultät OWL, \\ Universität Bielefeld \\ 2 Zentrum für Orthopädie und Unfallchirurgie, Universitäts- \\ medizin Mainz, Deutschland \\ 3 Klinik für Allgemein-, Viszeral- und Transplantations- \\ chirurgie, Universitätsmedizin Mainz, Deutschland \\ 4 Klinik und Poliklinik für Allgemein- und Abdominal- \\ chirurgie, Universitätsmedizin der Johannes Gutenberg- \\ Universität, Mainz, Deutschland \\ 5 Klinik für Allgemein- und Viszeralchirurgie, St. Georg \\ Klinikum Eisenach gGmbH, Deutschland
}

Sebastian Kuhn ${ }^{1,2 *}$ (D) , Florentine Huettl ${ }^{3 *}$, Kim Deutsch², Elisa Kirchgässner², Tobias Huber ${ }^{3}$, Werner Kneist ${ }^{4,5}$

\section{Schlüsselwörter}

digitale Transformation, Virtual/Augmented Reality, Robotik, computerassistierte Chirurgie, medizinische Ausbildung

\section{Key words}

digital transformation, virtual/augmented reality, robotic, computer-assisted surgery, medical education

\author{
Bibliografie \\ Zentralbl Chir 2021; 146: 37-43 \\ DOI $10.1055 / a-1265-7259$ \\ ISSN 0044-409X \\ (c) 2021. The Author(s). \\ This is an open access article published by Thieme under the terms of the Creative \\ Commons Attribution-NonDerivative-NonCommercial-License, permitting copying \\ and reproduction so long as the original work is given appropriate credit. Contents \\ may not be used for commercial purposes, or adapted, remixed, transformed or \\ built upon. (https://creativecommons.org/licenses/by-nc-nd/4.0/) \\ Georg Thieme Verlag KG, Rüdigerstraße 14, \\ 70469 Stuttgart, Germany \\ Korrespondenzadresse \\ Univ.-Prof. Dr. med. Sebastian Kuhn, MME \\ Professor für Digitale Medizin \\ Leiter der AG 4 - Digitale Medizin \\ Medizinische Fakultät OWL, Universität Bielefeld \\ Universitätsstraße 25, 33615 Bielefeld, Deutschland \\ Tel.: 0521/1 06-67977 \\ sebastian.kuhn@uni-bielefeld.de
}

\section{ZUSAMMENFASSUNG}

Hintergrund Die digitale Transformation der Medizin verändert den Beruf des Arztes. Augmented und Virtual Reality (AR/VR) und die Robotik erfahren einen zunehmenden Einsatz in unterschiedlichen klinischen Kontexten und bedürfen einer begleitenden Aus- und Weiterbildung, die bereits im Medizinstudium beginnen muss. Hier besteht aktuell eine große Diskrepanz zwischen dem hohen Bedarf und der Anzahl an wissenschaftlich überprüften Konzepten. Ziel der vorliegenden Arbeit war die Konzeptionierung und strukturierte Evaluation eines neu entwickelten Lern-/Lehrkonzepts zur digitalen Transformation mit Fokus auf die chirurgische Lehre.

Methoden 35 Studierende haben in 3 Kursen des BlendedLearning-Curriculums „Medizin im digitalen Zeitalter“ teilgenommen. Das 4. Modul dieses Kurses thematisiert Virtual Reality, Augmented Reality und Robotik in der Chirurgie. Es gliedert sich in die folgenden Kursteile: (1) immersive Simulation einer laparoskopischen Cholezystektomie, (2) leberchirurgische Operationsplanung mittels AR/VR, (3) Basisfertigkeiten am VR-Simulator für robotische Chirurgie, (4) kollaborative OP Planung im virtuellen Raum und (5) Expertengespräch. Nach Abschluss des Gesamtcurriculums erfolgte eine qualitative und quantitative Evaluation des Kurskonzepts mittels semistrukturierter Interviews sowie anhand von standardisierten Prä-post-Evaluationsfragebögen.

Ergebnisse Im qualitativen Auswertungsverfahren der Interviews wurden 79 Textaussagen 4 Schwerpunktkategorien zugewiesen. Den größten Anteil (35\%) nahmen hierbei Äußerungen zum „Expertengespräch“ ein, das von den Studierenden als elementarer Teil des Kurskonzepts gewertet wurde. Darüber hinaus empfanden die Studierenden den Kurs als horizonterweiterndes „Lernerlebnis“ (29\% der Aussagen) mit einem hohen „Praxisbezug“ (27\%). Die quantitative Studierendenevaluation zeigt eine positive Entwicklung für die Teilkompetenzen Wissen und Fertigkeiten sowie eine Tendenz zu einer positiven Haltung nach Kursabschluss.

Schlussfolgerung Die chirurgische Lehre ist zur Entwicklung digitaler Kompetenzen prädestiniert. Dabei muss die Ge-

gleichberechtigte Erstautoren 
schwindigkeit des Veränderungsprozesses der digitalen Transformation im chirurgischen Fachgebiet beachtet und im curricularen Konzept verankert werden.

\section{ABSTRACT}

Background The digital transformation of healthcare is changing the medical profession. Augmented/Virtual Reality (AR/VR) and robotics are being increasingly used in different clinical contexts and require supporting education and training, which must begin within the medical school. There is currently a large discrepancy between the high demand and the number of scientifically proven concepts. The aim of this thesis was the conceptual design and structured evaluation of a newly developed learning/teaching concept for the digital transformation of medicine, with a special focus on the influence of surgical teaching.

Methods Thirty-five students participated in three courses of the blended learning curriculum "Medicine in the digital age". The 4th module of this course deals with virtual reality, augmented reality and robotics in surgery. It is divided into the following course parts: (1) immersive surgery simulation of a laparoscopic cholecystectomy, (2) liver surgery planning using AR/VR, (3) basic skills on the VR simulator for robotic surgery, (4) collaborative surgery planning in virtual space and (5) expert discussion. After completing the overall curriculum, a qualitative and quantitative evaluation of the course concept was carried out by means of semi-structured interviews and standardised pre-/post-evaluation questionnaires. Results In the qualitative evaluation procedure of the interviews, 79 text statements were assigned to four main categories. The largest share (35\%) was taken up by statements on the "expert discussion", which the students consider to be an elementary part of the course concept. In addition, the students perceived the course as a horizon-widening "learning experience" ( $29 \%$ of the statements) with high "practical relevance" (27\%). The quantitative student evaluation shows a positive development in the three sub-competences knowledge, skills and attitude.

Conclusion Surgical teaching can be profitably used to develop digital skills. The speed of the change process of digital transformation in the surgical specialty must be considered. Curricular adaptation should be anchored in the course concept.

\section{Einleitung}

Die Digitalisierung der Gesellschaft und Wirtschaft hat die Medizin und damit die Chirurgie erreicht. Vor allem in der operativen Medizin gewinnt der Einsatz von Virtual und Augmented Reality (VR/AR) zunehmend an Bedeutung. Durch den Einsatz kommerziell verfügbarer VR-Simulatoren können Operationen simuliert und praktisch operative Fertigkeiten trainiert werden. Dadurch werden Lernkurven für videoendoskopisch und robotisch assistierte Operationen verkürzt und Leistungsniveaus kontrolliert [1]. Ferner ist ein präoperatives Warm-up möglich [2]. Eine Weiterentwicklung der herkömmlichen VR-Simulatoren durch eine Kombination mit einer VR-Brille erhöht die Immersion der Simulation, sodass der Anwender zunehmend in eine virtuelle Operationsumgebung eintauchen kann [3-5]. Auch intraoperativ werden die neuen Technologien eingesetzt. So werden präoperative Schnittbildgebungen nach 3-dimensionaler Rekonstruktion während einer Operation zur Navigation eingesetzt [6]. Die AR-Technologien werden es ermöglichen, patientenspezifische 3-D-Rekonstruktionen auf das jeweilige reale Organ oder Körperteil zu projizieren, um die Orientierung während der Eingriffe weiter zu optimieren $[7,8]$.

Dabei ist die frühzeitige Berücksichtigung im Rahmen der Ausund Weiterbildung essenziell $[9,10]$. Aufgewachsen als erste Generation der „Digital Natives“ bewegen sich heutige Studierende jedoch weiterhin auf der „Consumer Ebene“ [11]. Allein der dauernde Kontakt mit digitalen Medien qualifiziert den chirurgischen Nachwuchs nicht, sich relevante digitale berufsspezifische Handlungskompetenzen anzueignen. Die Integration digitaler Kompetenzen in die Medizinerausbildung gewinnt vor diesem Hintergrund zunehmend an Bedeutung, jedoch adressiert weder der Nationale Kompetenzbasierte Lernzielkatalog (NKLM) aus dem
Jahr 2015 noch der Masterplan Medizinstudium 2020 die Aspekte der digitalen Transformation [12,13].

Im Rahmen der vorliegenden Arbeit soll nun dargestellt werden, wie es innerhalb eines neu aufgesetzten Curriculums „Medizin im digitalen Zeitalter“ gelingen kann, die Entwicklungen im Bereich der „Virtual Reality, Augmented Reality und Robotik“ von chirurgischer Seite verantwortungsbewusst einzubringen. Modular werden die Lehrmethodik und der damit verbundene Lehrerfolg erstmals strukturiert evaluiert.

\section{Material und Methoden}

Seit dem Sommersemester 2017 wird an der Universitätsmedizin Mainz, beruhend auf der evidenzbasierten Systematik nach Kern, das modulare Blended-Learning-Curriculum „Medizin im digitalen Zeitalter angeboten“ $[14,15]$ ( $\triangleright$ Abb. 1).

\section{Studierende}

An den ersten 3 ausgewerteten Kursen haben 35 Studierende (24 männlich; 11 weiblich) der Medizin des 7.-9. Fachsemesters teilgenommen.

\section{Lehrende}

Das Modul 4 wurde von 4 chirurgischen Dozierenden betreut (3 männlich, 1 weiblich).

\section{Equipment}

Der Kurs fand in den Räumlichkeiten der „Rudolf Frey Lernklinik“ der Universitätsmedizin Mainz statt. Die digitale Simulationstechnik (Hard- und aktuelle Software) wurde von der chirurgischen Klinik zur Verfügung gestellt ( $\triangleright$ Tab. 1). 


\section{Medizin im digitalen Zeitalter - Module}

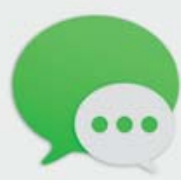

1

Digitale

Kommunikation

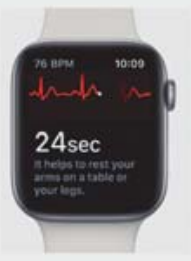

2

Apps \&

Smart Devices

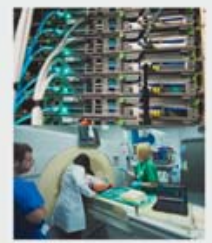

3

Telemedizin

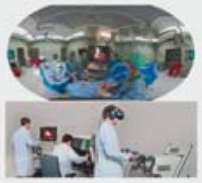

4

VR/AR \&

Robotik

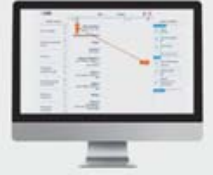

5

Künstliche Intelligenz

- Abb. 1 Einbettung des Modul 4 „VR, AR \& Robotik“ als Teil Blended-Learning-Curriculum „Medizin im digitalen Zeitalter“. Foto: Peter Pulkowski

> Tab. 1 Technische Geräte und Anwendungen im „Modul 4: Virtual Reality, Augmented Reality und computerassistierte Chirurgie“ des Curriculums „Medizin im digitalen Zeitalter“.

\begin{tabular}{|l|l|l|}
\hline VR-Laparoskopiesimulator & LapSim, Surgical Science (Göteborg, Sweden) & $\begin{array}{l}\text { - immersiver Operationssaal Allgemeinchirurgie (in } \\
\text { Kombination mit VR-Brille) }\end{array}$ \\
\hline VR-Robotiksimulator & $\begin{array}{l}\text { Mimic dV-Trainer, Mimic Technologies Inc. (Seattle, } \\
\text { WA, United States) }\end{array}$ & \begin{tabular}{l} 
- Basisfertigkeiten für robotische Chirurgie \\
\hline VR-Brille
\end{tabular} \\
\hline $\begin{array}{ll}\text { VIVE, HTC (Seattle, WA, United States) } \\
\text { VIVE Pro, HTC (Seattle, WA, United States) }\end{array}$ & $\begin{array}{l}\text { - immersiver Operationssaal Allgemeinchirurgie (in } \\
\text { Kombination mit VR-Simulator) }\end{array}$ \\
\hline AR-Brille & HoloLens, Microsoft (Redmond, WA, United States) & - OP-Planung Viszeralchirurgie (einzeln und kollaborativ) \\
\hline
\end{tabular}

\section{Vorbereitung}

In Analogie zu den Modulen 1-3 und 5 konnten sich die Studierenden auf das Modul 4 anhand eines E-Books vorbereiten [14, 16]. Für die Kurskommunikation in den E-Learning-Phasen kam das digitale Kommunikationstool (SLACK) zum Einsatz.

\section{Impulsvortrag}

Ein multimedialer Impulsvortrag mit klinischem Fallbeispiel führt in die Thematik VR, AR und Robotik ein ( $\bullet$ Video 1). Aktueller Stand der Forschung und Zukunftsvisionen werden vermittelt. Im Anschluss werden Lernziele und Erwartungen formuliert. Im Dialog mit den Dozierenden werden Fragen direkt beantwortet sowie auf das Lernen durch Erfahrung verwiesen und ggf. bereits Themen für das Interview formuliert.

\section{Dozierende als Lernbegleiter}

Im Kursverlauf nehmen alle 4 Dozierenden die Rolle des Lernbegleiters ein, die insbesondere in Zusammenhang mit dem Lehr-/ Lernkonzept des problemorientierten Lernens, bekannt ist [17]. In diesem Setting stehen die Studierenden im Mittelpunkt. Ziel ist die aktive und konstruktive Auseinandersetzung mit den Inhalten des Moduls 4, die anhand relevanter chirurgischer Beispiele vermittelt werden. Die Chirurgen fördern, moderieren und dosieren

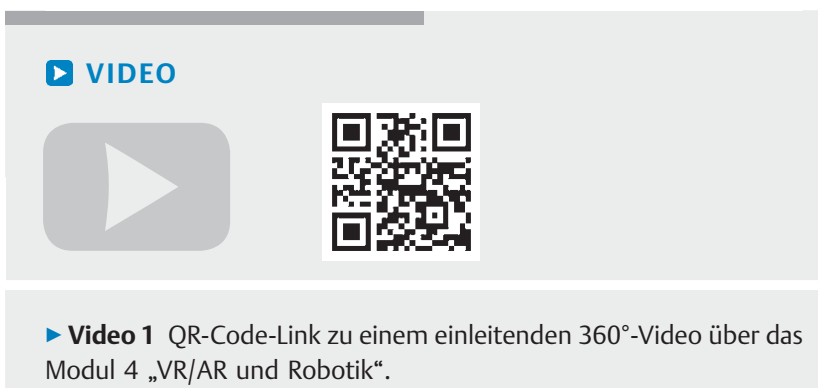

den Lernprozess und fungieren über den gesamten Zeitraum als Unterstützer einer selbstverantwortlichen Auseinandersetzung.

\section{Lernen durch Erfahrung}

Die praxisbezogene Interaktion umfasst verschieden VR-/ARTechnologien ( $\bullet$ Tab. 1) und bietet die Grundlage für die Kollaboration und Diskussion. Hier wird auf ein Lernen durch Erfahren gesetzt. Die Studierenden durchlaufen in 2er-Gruppen zunächst die neu entwickelte hochimmersive VR-Simulation einer minimalinvasiven Cholezystektomie. Dabei wird an einem Simulator die virtuelle Operation auf einem Monitor simuliert. Zusätzlich tauchen die Studierenden mithilfe einer VR-Brille in einen virtuellen Opera- 


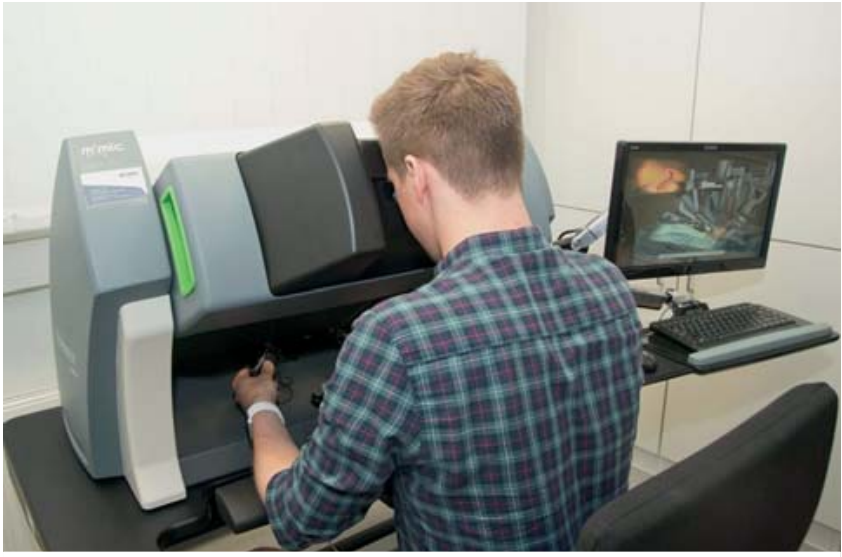

- Abb. 2 Übung zur Gefäßdissektion am virtuellen Simulator für robotische Chirurge (Mimic dV-Trainer, Mimic Technologies Inc, Seattle, USA). Foto: Peter Pulkowski

tionssaal ein [5]. Im Anschluss wird mithilfe eines VR- sowie eines AR-Headsets die Operation eines Lebertumors geplant. An einer 3. Station erproben die Teilnehmenden erste eigene Basisfertigkeiten am VR-Simulator für robotische Chirurgie ( $\sim$ Abb. 2). Fakultativ kann mit 2 VR-Brillen im Team am virtuellen Lebermodell studiert, aktiv gearbeitet und strategisch geplant werden ( $\triangleright$ Video 2).

\section{Experteninterview}

Das Experteninterview bietet den Studierenden die Möglichkeit, in 2er- oder 3er-Gruppen jeweils 20 min einen in der Thematik erfahrenen Viszeralchirurgen zu befragen. Die Fragen wurden im Vorfeld von den Studierenden erarbeitet und über SLACK ausgetauscht.

\section{Evaluation}

Die Evaluation erfolgte qualitativ in Form semistrukturierter Interviews und quantitativ anhand standardisierter Prä-post-Evaluationsfragebögen. Die quantitative deskriptive Evaluation wurde getrennt für die Teilkompetenzen „Wissen“, „Fertigkeiten“ und „Haltung“ anhand einer 7-stufigen Likert-Skala durchgeführt. Die Erhebung der qualitativen Evaluation erfolgte anhand eines teilstrukturierten Leitfadens. Hierbei wurden alle Teilnehmenden nach Abschluss des Gesamtcurriculums zu ihren subjektiven Erfahrungen von Projektmitarbeitern interviewt. Die Interviews umfassen ca. 45 min Sprechzeit pro Interview und bieten detaillierte Aussagen aus der Studierendenperspektive. Die „Qualitative Inhaltsanalyse nach Philipp Mayring“ wurde zur Analyse und Auswertung der im Rahmen der Evaluation erhobenen und transkribierten Interviews genutzt $[18,19]$. Dabei werden die Aussagen schrittweise in spezifische Kategorien eingeteilt ( $\triangleright$ Tab. 2). Durch Beachtung der Sinnzusammenhänge, Wertungen und Relevanzen wurde der für das Modul 4 spezifische Textanteil bestimmt ( $\bullet$ Abb. 3). Die quantitative Evaluation zum subjektiven Lernerfolg wurde für die Teilkompetenzen „Wissen“ und „Fertigkeiten“ in Form einer Vorher-nachher-Selbsteinstufung mit einer 7-stufigen
- Tab. 2 Kategorientabelle „Virtual Reality, Augmented Reality und Robotik“ zur qualitativen Analyse der semistrukturierten Interviews.

\begin{tabular}{|c|c|}
\hline $\begin{array}{l}\text { Lern- } \\
\text { erlebnis }\end{array}$ & $\begin{array}{l}\text { - erstmaliger Kontakt mit AR/VR und robotischer } \\
\text { Chirurgie } \\
\text { - Beschreibung der Lernerfolge } \\
\text { - in Verhältnis setzen zu „regulärer“ Lehre }\end{array}$ \\
\hline $\begin{array}{l}\text { Praxis- } \\
\text { bezug }\end{array}$ & $\begin{array}{l}\text { - immersive realitätsnahe Erfahrung } \\
\text { - Skills-Training } \\
\text { - Theorie-Praxis-Reflexion }\end{array}$ \\
\hline $\begin{array}{l}\text { Experten- } \\
\text { gespräch }\end{array}$ & $\begin{array}{l}\text { - direkter Austausch mit Experten als Rollenmodell } \\
\text { - Festigung der Praxiserfahrung im Dialog } \\
\text { - empfundene Wertschätzung }\end{array}$ \\
\hline $\begin{array}{l}\text { Digital } \\
\text { Literacy }\end{array}$ & $\begin{array}{l}\text { - Ausformulierung einer persönlichen Haltung } \\
\text { - Selbstreflexion/Reflexion des ärztlichen Berufsbildes } \\
\text { - Bezug zur gesellschaftlichen Ebene }\end{array}$ \\
\hline
\end{tabular}

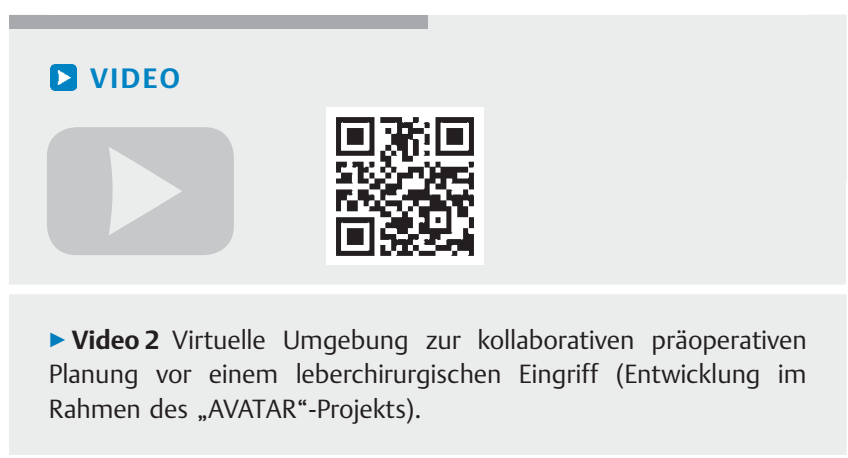

Likert-Skala ( 1 = sehr gering bis 7 = sehr hoch) vorgenommen. Zusätzlich wurde die Einschätzung der persönlichen „Haltung“ (1 = sehr negativ, 4 = neutral, 7 = sehr positiv) abgefragt.

\section{Ergebnisse}

\section{Qualitative Evaluation anhand semistrukturierter Interviews}

Die Evaluation in Form der semistrukturierten Interviews zeigt die hohe Akzeptanz des Kurskonzepts. Die Studierenden betonen dabei die empfundene Wertschätzung und Motivation durch die intensive und kreative Zusammenarbeit untereinander und mit den Chirurgen. Besonders die Möglichkeit der praktisch-operativen Interaktion und Verdeutlichung von medizinischen Versorgungskonzepten wurden dabei positiv bewertet.

Im Auswertungsverfahren der Interviews wurden 79 Textaussagen der Kategorie „Virtual Reality, Augmented Reality und Robotik" zugeordnet ( $\triangleright$ Abb. 2). Davon fallen $29 \%$ in die Kategorie „Lernerlebnis“. Diese Kategorie beschreibt den erstmaligen Kontakt der Studierenden mit hochdigitalisierter Medizintechnik. Die Befragten sprechen von einem horizonterweiternden Erlebnis. Hier schließt sich auch die Kategorie „praktische Handlungserfahrung“ mit 27\% der Aussagen an. Diese Kategorie umfasst die tatsächlichen Lernerfolge, vor allem durch das praktische Ausprobieren der Technik sowie die in Gespräch und Diskussion angestoßene Reflexion. Das „Expertengespräch“, eine für sich stehen- 


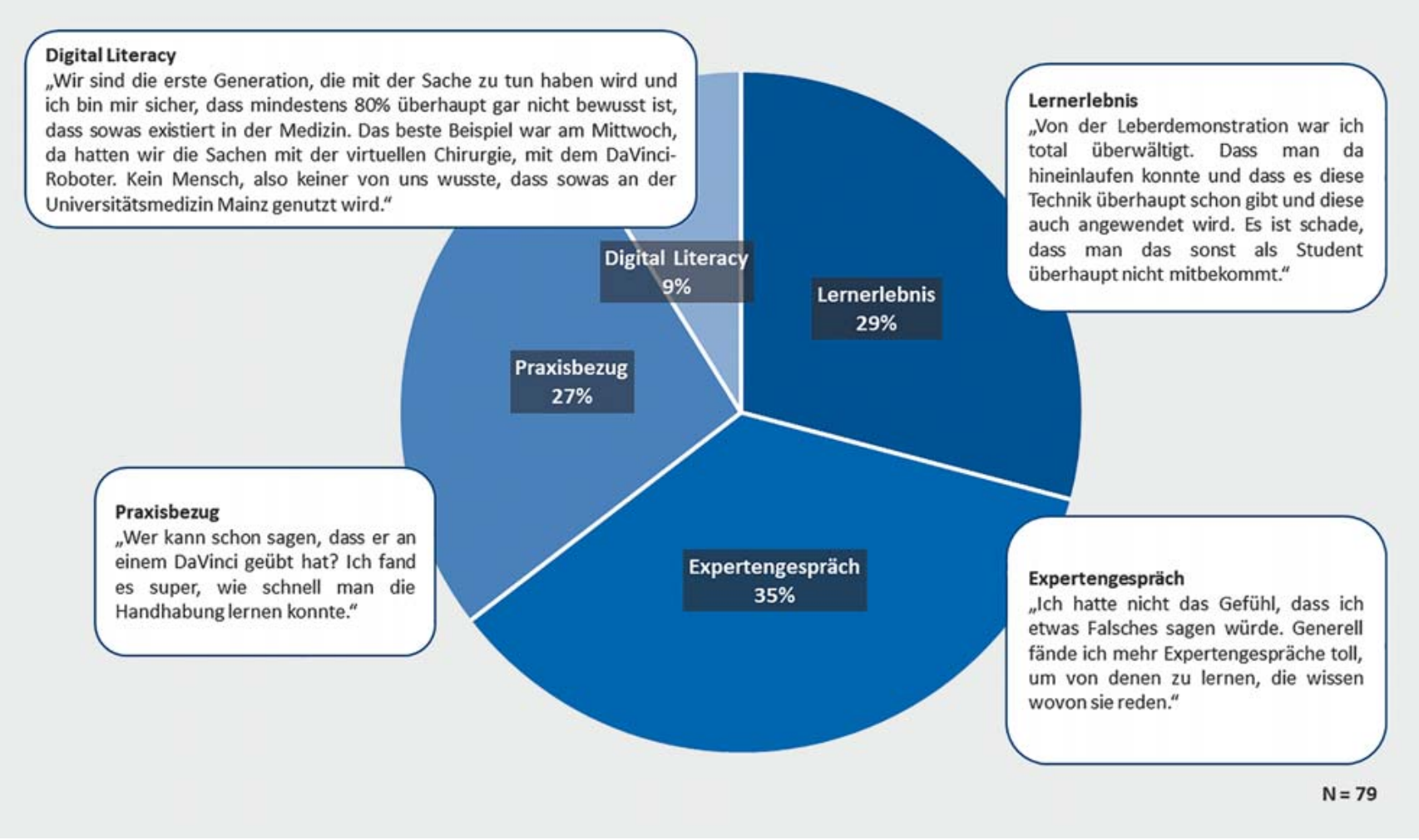

Abb. 3 Ergebnisse der qualitativen Evaluation anhand semistrukturierter Interviews. Anteile der 4 Kategorien mit exemplarischen Zitaten der Studierenden

de Kategorie, wurde durch die Studierenden als elementarer Teil des Kurskonzepts gewertet. Die Möglichkeit des direkten Austauschs mit einem in der computerassistierten Chirurgie erfahrenen Operateur trägt dazu bei, die erlebte Praxiserfahrung zu festigen. Die Studierenden betonen hierbei den Austausch über professionelles chirurgisches Handeln mit einem Rollenmodell als seltene Möglichkeit im Studium und schätzen diese als sehr positiv ein. Dies führt zu hohem Lernerfolg und zu einer nachhaltigen differenzierten Haltung gegenüber VR/AR und Robotik. 35\% der getroffenen Aussagen können dieser Kategorie zugeordnet werden. Sowohl die beschriebene Möglichkeit zum Gespräch als auch die Möglichkeit, an und mit neuer Simulatortechnik zu üben, wird von den Studierenden sehr positiv aufgefasst und mit empfundener Wertschätzung sowie hoher intrinsischer Motivation verbunden. Die Kategorie „Digital Literacy“ beschreibt mit 9\% der Aussagen eine ganzheitliche Auseinandersetzung mit den digitalen Fertigkeiten hinsichtlich eines kritischen, planvollen und kontextspezifischen Umgangs mit zur Verfügung stehenden Daten [20]. Verbesserungsvorschläge der Studierenden betreffen vor allem den zeitlichen Rahmen. Die Teilnehmenden erachten es als sinnvoll, längere Unterrichtseinheiten zu planen, um mehr Zeit für Diskussionen, Expertengespräche und Praxisübungen zu schaffen. Des Weiteren äußern einige Teilnehmende den Wunsch, dass die „hands-on“-Anteile weiter erhöht werden, bspw. um die Planungssoftware detaillierter austesten zu können.

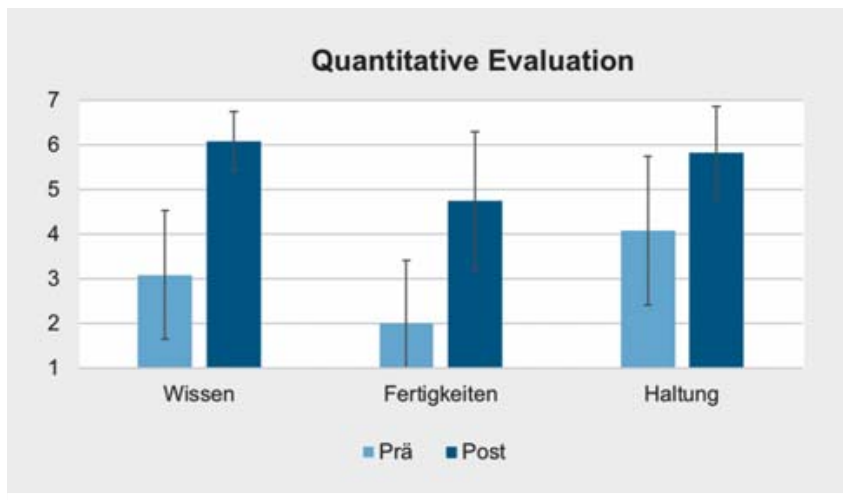

- Abb. 4 Ergebnisse des quantitativen Prä-post-Vergleichs der Bereiche „Wissen“, „Fertigkeiten“ und „Haltung“ ( 1 = sehr gering, 4 = neutral, 7 = sehr hoch) und „Haltung“ ( 1 = sehr negativ, 4 = neutral, $7=$ sehr positiv) zu Virtual Reality, Augmented Reality und Robotik.

\section{Quantitative Prä-post-Evaluation}

Die Prä-post-Evaluation zeigt eine positive Selbsteinschätzung des Lernerfolgs in den Teilkompetenzen Wissen (vorher: 3,08 $\pm 1,44$; nachher: 6,08 $\pm 0,67$ ) und Fertigkeiten (vorher: 2,00 $\pm 1,41$; nachher $4,75 \pm 1,54)$. Die Haltung der Teilnehmenden wurde vom neutralen in den positiven Bereich (vorher: 4,08 $\pm 1,68$; nachher $5,83 \pm 1,03$ ) verschoben ( $\triangleright$ Abb. 4 ). 


\section{Diskussion}

Im Gesundheitssektor werden VR- und AR-Technologien in der Prävention, der Rehabilitation (psychologische Therapie), im Produktmarketing und in der Unternehmenspräsentation eingesetzt. Der Chirurg und Militärpilot R. Satava hat schon 1993 die immense Bedeutung der Simulation für die zukünftige chirurgische Ausbildung, die Schulung und das Training erkannt [21]. Im Jahr 2016 sieht das Hochschulforum Digitalisierung VR und AR als Schlüsseltechnologien, welche die Weiterentwicklung der Hochschulbildung beeinflussen. Der Reifegrad für den Durchbruch sei erreicht [22]. Mit der vorliegenden Arbeit wollen wir einen Beitrag leisten, um dieses Postulat im Rahmen eines Unterrichtskonzepts des Medizinstudiums strukturiert zu evaluieren.

35 Studierende der Humanmedizin experimentieren im Modul 4 des standardisierten Curriculums „Medizin im digitalen Zeitalter" und erweitern ihr Verständnis für Anatomie, Topografie, Simulation, Navigation und computerassistierte Chirurgie. Eindrucksvoll wird von einem Viertel der Studierenden betont, dass kontextuelle Lernerlebnisse möglich sind. Dies ist ein zunehmend wichtiger Aspekt, weil Studierende sehr frühzeitig vom Fach Chirurgie überzeugt werden müssen [23]. Nach Umfrageergebnissen verfügen nur etwa $30 \%$ der deutschen Universitätskliniken über Simulatoren zur VR-Simulation und es ist weitgehend unklar, ob sie in der studentischen Lehre curricular eingesetzt werden [24]. Das vorliegend beschriebene curriculare Skills-Training in der virtuellen Realität wird mit seinem unmittelbaren Praxisbezug in 29\% der Interviews positiv herausgestellt. Die aktuelle Literaturanalyse belegt den Nutzten von VR- und Box-Trainern zur Verbesserung der Aus- und Weiterbildung und stellt hier eine Überlegenheit gegenüber reinen Videotrainern (oder auch „dry laboratory simulation“) fest [24-26].

Verglichen mit traditioneller Lehre oder Blended Learning verbessern AR- und VR-Simulationsszenarien den Wissensstand und die kognitiven Fähigkeiten der Teilnehmer in höherem Maße [27]. Dass die beschriebene modulare chirurgische Ausbildung das digitale Profil der Medizinstudierenden verstärkt, kann nun mit der sehr guten qualitativen Evaluation sowie dem subjektiven Lernerfolg anhand der quantitativen Evaluation gezeigt werden. Eine klare Limitation besteht zum jetzigen Zeitpunkt hinsichtlich des hohen Aufwands aufgrund der technischen Ausstattung und personalintensiven Umsetzung aufgrund der praktischen Anteile.

Die Digitalisierung der Chirurgie und der gesamten Medizin ist ein fundamentaler Wandlungsprozess, dessen Einfluss auf das zukünftige Berufsbild des Arztes noch nicht abgeschätzt werden kann. Jedoch steht fest, dass eine begleitende Ausbildung erforderlich ist [14]. Unbedingt müssen in Zukunft Szenarien, die mehr oder weniger differenziert vom Ersatz des chirurgischen Berufs durch künstliche Intelligenz und Robotik ausgehen, von den Studierenden hinterfragt, aber vor allem aus der Praxiserfahrung heraus mit Experten analog diskutiert werden. Durch diesen Dialog und in Kombination mit den Handlungserfahrungen aus der VR/ AR wird ein weiterer Schwerpunkt des Curriculums „Medizin im digitalen Zeitalter“ gestärkt - die Vermittlung einer Haltung.

Die Reflexion der Haltung gegenüber neuen digitalen Technologien und Medien (VR/AR) und dem weiten Themenkomplex des digitalen Wandels (Künstliche Intelligenz, Datenschutz, Ethik) spielt eine wichtige Rolle. Dieser Aspekt wird nach den vorliegenden Ergebnissen zu einem zentralen Lernmoment. Die Studierenden können sich mit der Aktualität und Realität der technischen Entwicklungen inklusive progressiver Expertisen auseinandersetzen $[28,29]$. Sie bekommen so einen neuen Zugang zur Materie. Der chirurgische Lehrer nimmt dabei die Rolle des Lernbegleiters ein. Durch den Erfahrungsaustausch in der Peergroup sowie mit Experten wird eine reflexive Auseinandersetzung mit der digitalen Thematik gefördert. Das praktische Eingreifen der Studierenden in das Geschehen bietet die Möglichkeit, Lernende aktiv in die Ausbildung einzubinden und Wissensinhalte virtuell im entsprechenden situativen und sozialen Kontext zu erleben. In weiterentwickelten Konzepten zur kollaborativen Zusammenarbeit über Distanzen können mithilfe der gewonnen Erfahrung aus der direkten Interaktion mit den Studierenden neue Lehransätze exploriert werden (Video 2). Dabei ist die Kombination von quantitativer und qualitativer Evaluation nach iterativem Prinzip Voraussetzung, um agil und genau die Bedürfnisse sowohl der Studierenden als auch der Dozierenden an die Rahmenbedingungen anzupassen.

Hinsichtlich der qualitativen Evaluationsmethode lässt sich festhalten, dass sich diese insbesondere für Kurskonzepte eignet, die problemzentriertes Lernen in den Vordergrund stellen, da die Interviews auch den (kritischen) Austausch, die Positionierung und das Einordnen der Lehreinheiten in den individuellen Erfahrungskontext ermöglichen. Ihre Grenzen findet die Methode in der intersubjektiven Vergleichbarkeit bzw. der objektiven Messung von Lernfortschritten. Des Weiteren ist zu beachten, dass die Studierenden sich im Rahmen der Wahlpflichtwoche bewusst für die Teilnahme am Kurskonzept entscheiden und so davon ausgegangen werden kann, dass zumindest ein gewisses Interesse an der Thematik schon vorab bestanden hat.

Zusammenfassend wird ein sehr zeitgemäßes, VR- und ARbasiertes, modulares Lehr-Lern-Setting vorgestellt. Die positiven Veränderungen in der Kompetenzbeurteilung - Wissen, Fertigkeiten und Haltung - spiegeln dabei die bisherigen und neu erworbenen Erfahrungen der studentischen Kursteilnehmer wider. Bei der Entwicklung derartiger Curricula muss die enorme Geschwindigkeit des digitalen Veränderungsprozesses berücksichtigt und eine agile iterative Anpassung vorgenommen werden [30 - 32]. Die Integration dieser „chirurgisch“ vermittelten digitalen Kompetenzen in das Curriculum des Medizinstudiums ist somit nicht nur eine aktuelle, sondern auch eine fortwährende Herausforderung.

Danksagung

Medizin im digitalen Zeitalter wird als curriculares Reformprojekt der Universitätsmedizin Mainz vom Stifterverband im Rahmen des mit der Carl-Zeiss-Stiftung gemeinsam initiierten Programms „Curriculum 4.0“ gefördert. Die verwendeten Simulatoren Laparoskopie und Robotik wurden durch inneruniversitäre Forschungsförderung der JohannesGutenberg-Universität und durch 2 Förderprojekte für medizinische Lehre der Universitätsmedizin Mainz („MAICUM“) gefördert. Das BMBFVerbundprojekt „AVATAR“ (BMBF FKZ 16SV0857) fördert die Entwicklung von augmentierten und virtuellen Multi-User-Anwendungen für den medizinisch-technischen Austausch in immersiven Räumen und unterstützt das Projekt durch Augmented-/Virtual-Reality Hard- und Software. 
Interessenkonflikt

Die Autorinnen/Autoren geben an, dass kein Interessenkonflikt besteht.

Literatur

[1] Gurusamy KS, Aggarwal R, Palanivelu L et al. Virtual reality training for surgical trainees in laparoscopic surgery. Cochrane Database Syst Rev 2009. doi:10.1002/14651858.CD006575.pub2

[2] Pike TW, Pathak S, Mushtaq F et al. A systematic examination of preoperative surgery warm-up routines. Surg Endosc 2017; 31: 2202-2214

[3] Huber T, Paschold M, Hansen C et al. „Ich laparoskopier' dann woanders weiter". Chirurg 2017; 88: 956-960

[4] Huber T, Wunderling T, Paschold M et al. Highly immersive virtual reality laparoscopy simulation: development and future aspects. Int I Comput Assist Radiol Surg 2018; 13: 281-290

[5] Huber T, Paschold M, Hansen C et al. New dimensions in surgical training: immersive virtual reality laparoscopic simulation exhilarates surgical staff. Surg Endosc 2017; 31: 4472-4477

[6] Oldhafer K], Peterhans M, Kantas A et al. [Navigated liver surgery: Current state and importance in the future]. Chirurg 2018; 89: 769-776

[7] Queisner M, Pratschke J, Sauer IM. Response: ,Mixed and Augmented Reality-Why Surgeons Should Care'. Ann Surg 2018. doi:10.1097/ sla. 0000000000002913

[8] Cho J, Rahimpour S, Cutler A et al. Enhancing Reality: A Systematic Review of Augmented Reality in Neuronavigation and Education. World Neurosurg 2020; 139: 186-195. doi:10.1016/j.wneu.2020.04.043

[9] Kirschniak A, Egberts JH, Granderath FA et al. Augmented Reality, CyberPhysical Systems and Robotic Surgery: Nice to Have or a Program with Future? Visc Med 2018; 34: 60-65

[10] Zhao J, Xu X, Jiang H, Ding Y. The effectiveness of virtual reality-based technology on anatomy teaching: a meta-analysis of randomized controlled studies. BMC Med Educ 2020; 20: 127. doi:10.1186/s12909020-1994-z

[11] Persike M, Friedrich J-D. Lernen mit digitalen Medien aus Studierendenperspektive. Sonderauswertung aus dem CHE Hochschulranking für die deutschen Hochschulen. Arbeitspapier Nr. 17. Berlin: Hochschulforum Digitalisierung; 2016

[12] Bundesministerium für Bildung und Forschung. Masterplan Medizinstudium 2020. Im Internet (Stand: 28.06.2018): http://www.bmbf.de/de/ masterplan-medizinstudium-2020-4024.html

[13] Medizinischer Fakultätentag der Bundesrepublik Deutschland e.V. Nationaler Kompetenzbasierter Lernzielkatalog Medizin 2015 (NKLM). Im Internet (Stand: 09.11.2020): http://www.nklm.de/kataloge/nklm/ lernziel/uebersicht

[14] Kuhn S. Medizin im digitalen Zeitalter: Transformation durch Bildung. Dtsch Arztebl 2018; 115: A633-A638

[15] Kuhn S, Jungmann F. Medizin im digitalen Zeitalter. Radiologe 2018; 58 : 236-240
[16] Kuhn S, Kirchgässner E, Deutsch K. Medizin im digitalen Zeitalter - „Do it by the book ... but be the author!". Synergie 2017. Im Internet (Stand: 09.11.2020): https://www.synergie.uni-hamburg.de/de/media/ausgabe04/synergie04.pdf

[17] Strittmatter-Haubold V. Problem Based Learning. Eine Methode für wirksame Lernsettings in der wissenschaftlichen Weiterbildung? Hochschule und Weiterbildung 2016; 1: 50-55

[18] Mayring P. Qualitative Content Analysis. In: Flick U, von Kardoff E, Steinke I, eds. A Companion to Qualitative Research. Glasgow, UK: SAGE; 2004: 266-269

[19] Mayring P. Qualitative Inhaltsanalyse. In: Mey G, Mruck K, Hrsg. Handbuch Qualitative Forschung in der Psychologie. Weinheim: VS Verlag für Sozialwissenschaften; 2010. doi:10.1007/978-3-531-92052-8_42

[20] Kuhn S, Kadioglu D, Deutsch K et al. Data Literacy in der Medizin. Onkologe 2018; 24: 368-377

[21] Satava RM. Virtual reality surgical simulator. The first steps. Surg Endosc 1993; 7: 203-205

[22] Hochschulforum Digitalisierung. The Digital Turn - Hochschulbildung im digitalen Zeitalter. Arbeitspapier Nr. 27. Berlin: Hochschulforum Digitalisierung; 2016

[23] Ghadimi M. Editorial. Zentralbl Chir 2019; 144: 137-138

[24] Huber T, Kirschniak A, Johannink J. Umfrage zum Training laparoskopischer Fertigkeiten in Deutschland. Zentralbl Chir 2017; 142: 67-71

[25] Bric JD, Lumbard DC, Frelich MJ et al. Current state of virtual reality simulation in robotic surgery training: a review. Surg Endosc 2016; 30: 2169-2178

[26] Alaker M, Wynn GR, Arulampalam T. Virtual reality training in laparoscopic surgery: A systematic review \& meta-analysis. Int J Surg 2016; 29: 85-94

[27] Kyaw BM, Saxena N, Posadzki P et al. Virtual Reality for Health Professions Education: Systematic Review and Meta-Analysis by the Digital Health Education Collaboration. J Med Internet Res 2019; 21: e12959

[28] Hung AJ, Chen J, Gill IS. Automated Performance Metrics and Machine Learning Algorithms to Measure Surgeon Performance and Anticipate Clinical Outcomes in Robotic Surgery. JAMA Surg 2018; 153: 770-771

[29] Grace K, Salvatier J, Dafoe A et al. When will Al exceed human performance? Evidence from Al experts. J Artif Intell Res 2018; 62: 729-754

[30] Baumgartner P, Brei C, Gerdes A et al. Curriculumentwicklung und Kompetenzen für das digitale Zeitalter - Thesen und Empfehlungen der AG Curriculum 4.0 des Hochschulforum Digitalisierung. Arbeitspapier Nr. 39. Berlin: Hochschulforum Digitalisierung; 2018

[31] Michel A, Baumgartner P, Brei $C$ et al. Framework zur Entwicklung von Curricula im Zeitalter der digitalen Transformation. Diskussionspapier Nr. 01 (Version 2.0). Berlin: Hochschulforum Digitalisierung; 2018

[32] Hallersleben S, Loewe A; Praxisnetz Digitale Technologien. Szenario zur Leitfrage „Wie wirken Arzt und digitale Technik im Jahr 2035 zusammen?“. VDE/DGBMT 2019. Im Internet (Stand: 09.11.2020): https:// www.vde.com/resource/blob/1911830/4258d19eabba0aa39dcfffef 96005ed2/szenario-health---download-hb-data.pdf 